\title{
Datenbasierte Pandemie-Planung in einem Spitalverbund
}

\section{Thomas Kaufmann a,b,f, Guido Schüpfer ${ }^{a, c, f}$, Lukas Fieber ${ }^{d, f}$, Christoph Konrad $^{e, f}$}

${ }^{a}$ Dr. med.; ${ }^{b}$ OP Management; ${ }^{c}$ MBA PhD, Stab Medizin und Klinik für Anästhesie; ${ }^{d}$ cand. med., Klinik für Anästhesie; ${ }^{e}$ Prof. Dr. med., Departement für Klinische Querschnittsmedizin und Klinik für Anästhesie; ${ }^{\dagger}$ Luzerner Kantonsspital

Es ist herausfordernd, angemessene Entscheidungen in einem mit viel Unsicherheit behafteten dynamischen Umfeld zu treffen. In Zeiten der Covid-Pandemie und knappen Intensivkapazitäten ist eine sinnvolle Verteilung der Ressourcen essentiell [1]. Wir stellen daher unsere Managementwerkzeuge vor, die sich bei uns zur Bewältigung dieser Herausforderung bewährt haben (Stand November 2020).

Das Luzerner Kantonsspital ist ein Klinikverbund mit zwei Regionalversorgern und einem Maximalversorger am Standort Luzern mit total 1000 Betten. In der Pandemiesituation wird der Verbund als Einheit aus einem strategischen Pandemiestab geführt. Die intensivmedizinischen Kapazitäten werden seitens der Intensivmedizin-Leitung in Abstimmung mit dem
Pandemiestab in der gesamten Zentralschweiz koordiniert. Ein abgestuftes, ampelbasiertes Kapazitätsmodell (Grün/Gelb/Rot entsprechen vordefinierten, konzernangepassten Auslastungsstufen) dient als Entscheidungsgrundlage für die koordinierten Änderungen im operativen Betrieb aller angeschlossenen Häuser. Die Anlehnung an eine Ampel erleichtert das

\section{Personalübersicht Rettungsdienst}

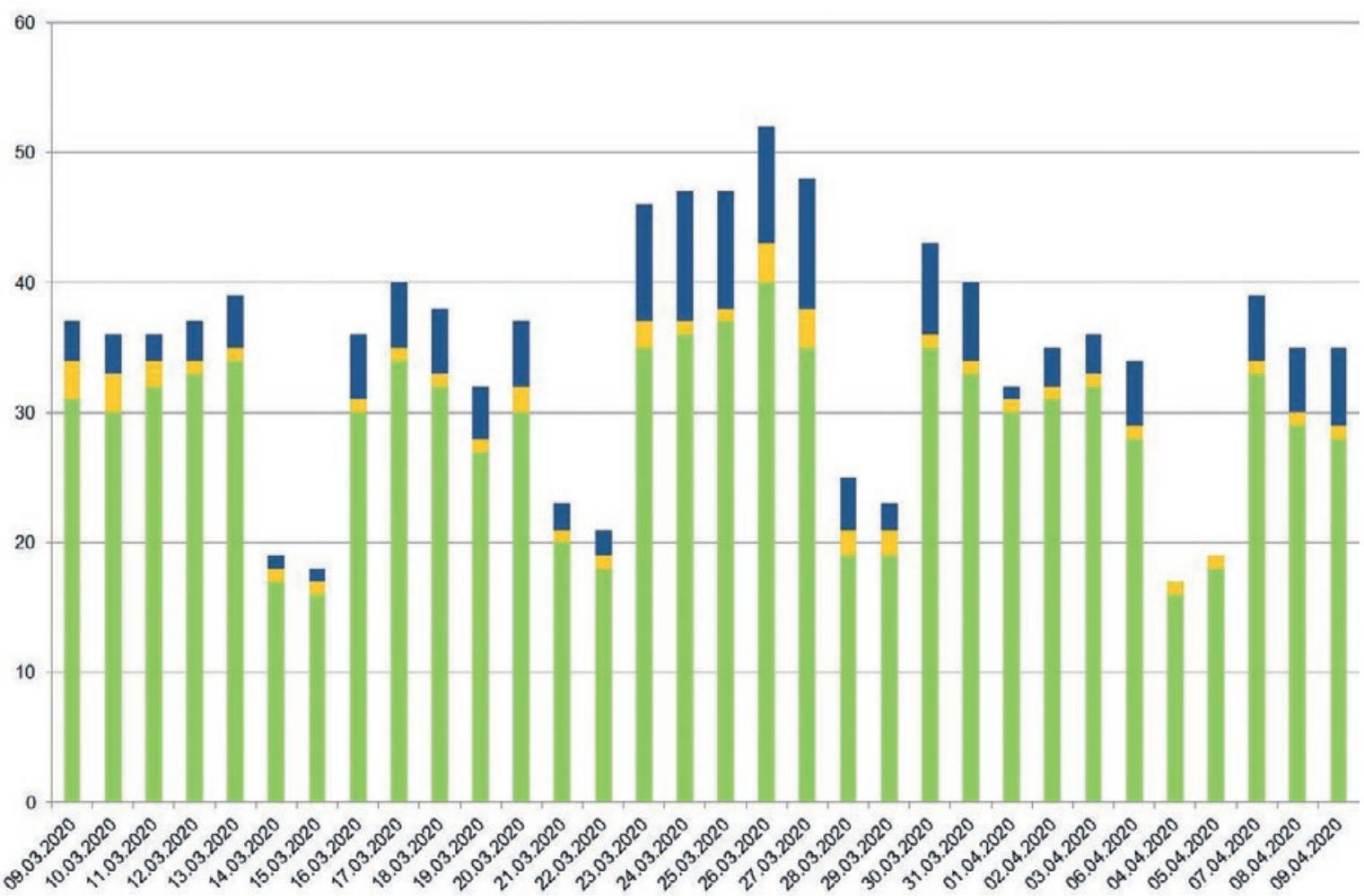

Abbildung 1: Die Säulen stellen die Anzahl der theoretisch verfügbaren Mitarbeitenden dar. 
interne Verständnis für die Einschätzung der betrieblichen Situation auf jeder Personalebene.

Verfügbare epidemiologische Daten beschrieben bis zum jetzigen Zeitpunkt meistens nationale oder überregionale Entwicklungen. Mathematische Modelle versuchen diese Entwicklungen zu prognostizieren, können aber meist nur den Verlauf der Erkrankungsprävalenz und nicht die Systembelastung beschreiben. Wir etablierten daher ein Monitoring-System im Sinne eines Frühwarnsystems für unseren eigenen Behandlungsablauf mit dem Ziel, mittelbar klinische und strategische Entscheidungen zu erleichtern. Dabei wurde auf die am meisten exponierten Bereiche eines klinischen Versorgers fokussiert [2] (präklinisches und klinisches Notfallsystem, OP, Anästhesie sowie Intensivmedizin). Zusätzlich stellen wir die Covid-Patienten im Behandlungssystem graphisch dar, abgestuft nach Versorgungsstufe, sowie krankheitsbedingte Ausfälle beim Personal. Die gewonnenen Daten werden in aggregierter Form allen Mitarbeiterinnen und Mitarbeitern regelmässig zur Verfügung gestellt. Damit es nicht zum Effekt der geteilten Aufmerksamkeit kommt, werden Themeninhalte in einzelnen Graphiken dargestellt [3]. Die Darstellung lehnte sich jeweils an bisher erstellte Berichte an, so dass es regelmässig in der gleichen Weise zur Informationsweitergabe kommt [4]. In diesem Paper wollen wir kurz diese Berichte beschreiben, deren graphische Auswertung präsentieren, um so möglicherweise anderen Kliniken einen Anhalt zu bieten und das Verständnis der laufenden Corona-Pandemie zu erleichtern.

\section{Personal}

Das Corona-Virus bedroht das eigene Personal, so dass es notwendig ist, frühzeitig zu erkennen, ob Krankheitsfälle zunehmen und damit die Behandlungskapazität abnimmt.

Wir erfassen auf Tagesbasis die An- und Abwesenheit des Personals des ärztlichen Dienstes, der Pflege und des Rettungsdienstes, im Notfallzentrum, in der Anästhesie, im OP und auf der Intensivstation. Hierbei werden Daten über anwesende Kolleginnen und Kollegen, erkranktes und mobilisierbares Personal erfasst und graphisch dargestellt.

Ziel ist es, frühzeitig Betriebsstörungen durch Abwesenheit von Personal zu erfassen. Es zeigte sich zu Beginn der Pandemie ein Erkrankungscluster im Bereich des Rettungsdienstes, das auf lokale Gegebenheiten im Arbeitsumfeld zurückzuführen war (zu kleine Sozialräume). Basierend darauf wurden organisatorische Änderungen, wie beispielsweise Pausenregelungen, für den gesamten Konzern getroffen und das Risiko im weiteren Verlauf für alle Mitarbeitenden reduziert. Mittlerweile werden die meisten Erkrankungen jedoch im privaten und nicht im beruflichen Umfeld der Mitarbeiter lokalisiert (siehe Abb. 1).

\section{OP-Management}

Das OP-Management ist die Organisationseinheit, in der der OP-Betrieb des Zentrumsspitals im Regelbetrieb zusammengefasst ist. Im Rahmen der Reduktion des

\section{Übersicht über genutzte OP-Kapazitäten im Konzern}

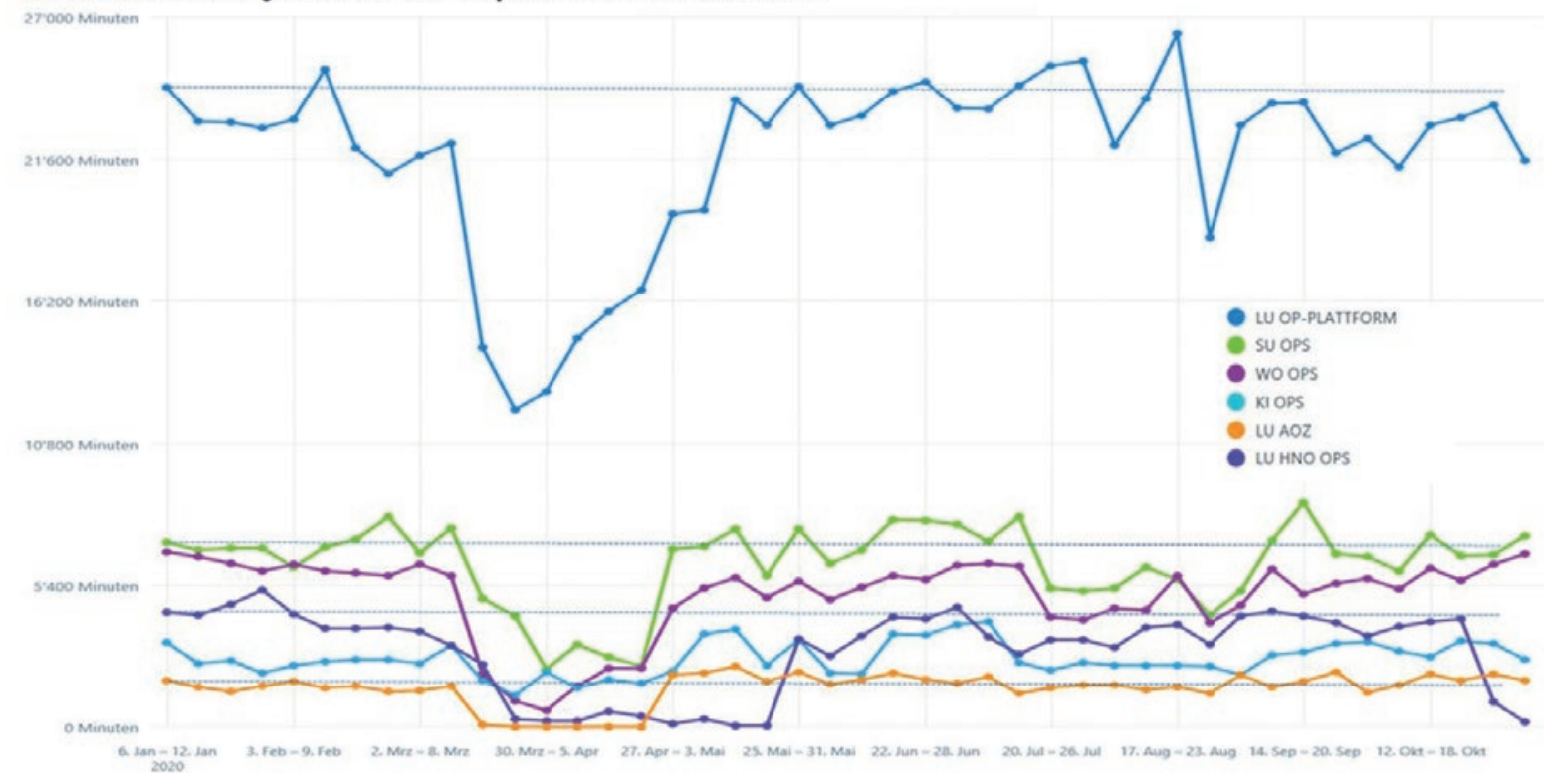

Abbildung 2: Die jeweilige OP-Zahl an verschiedenen Standorten (LU = Luzern; Su = Sursee; Wo = Wolhusen; KI = Kinderspital; $\mathrm{AOZ}=$ Ambulantes Operationszentrum). 


\section{Anzahl Covid-Patientinnen und -Patienten}

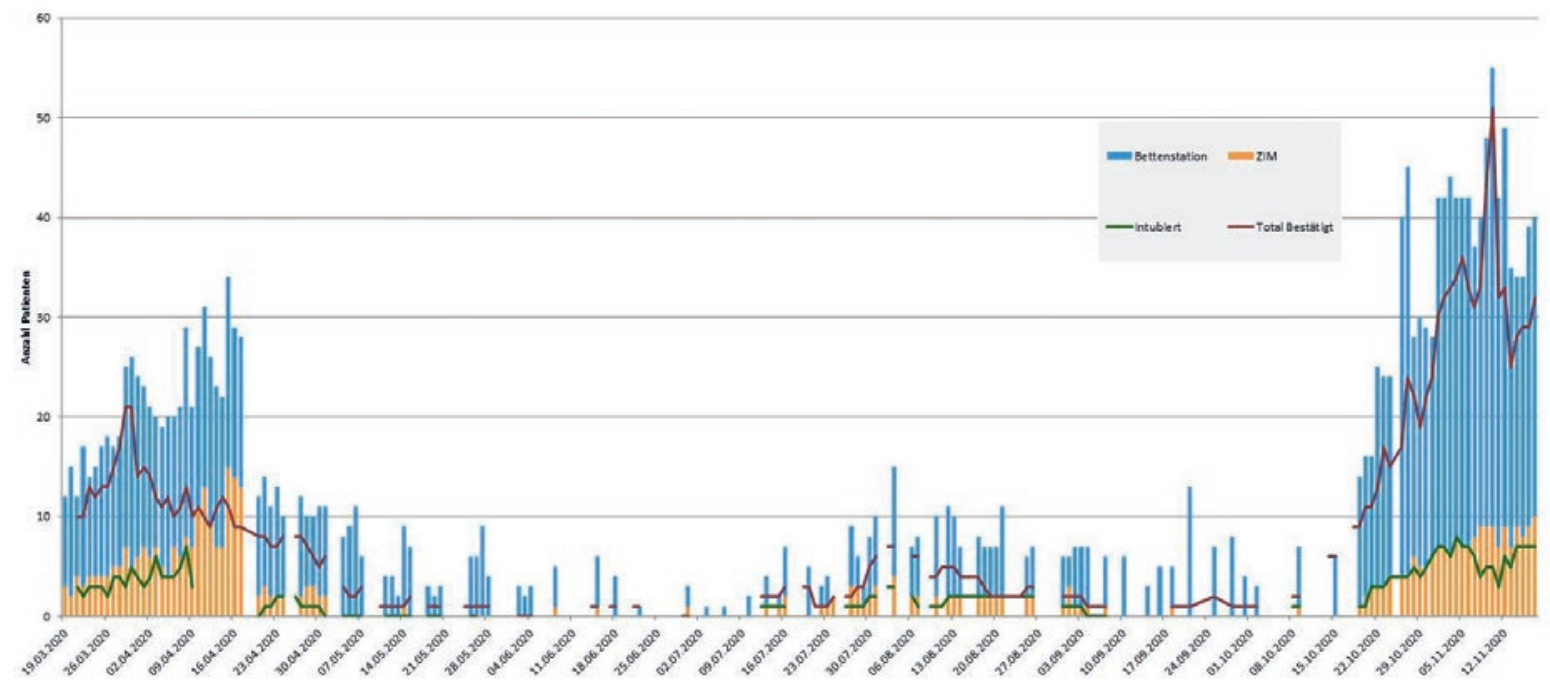

Abbildung 3: Covid-Fälle am Luzerner Kantonsspital, ohne Kinderspital. Eine Säule entspricht einem Tag (ZIM: Zentrum für Intensivmedizin).

elektiven Programms erfassen wir die Auslastung im OP-Bereich. Alle operativen Disziplinen ausser Kinderchirurgie, Kaiserschnittentbindungen, In-vitro-Fertilisation, Herzkatheterlabor sowie Augenklinik werden zentral betrieben. Nach der Indikationsstellung der jeweiligen operativen Disziplinen werden diese nochmals von dem für das OP-Programm verantwortlichen Chirurgen geprüft und final freigegeben. Nach einer Schwankung im Rahmen des ersten Lockdowns (März 2020) stabilisierte sich der OP-Prozess gemessen an der Schnitt-Naht-Zeit schnell binnen weniger Tage und wies eine Auslastung von circa 70\% im November im Vergleich zum Regelbetrieb des Vorjahres auf. Die Schnitt-Nahtzeit stellt die Dauer einer Operation oder Intervention vom Beginn bis zum Ende dar und ist für uns ein valides Mass für die Produktivität. Mittels dieser Zeit können unterschiedliche operative Disziplinen wie auch unterschiedliche Standorte miteinander verglichen werden.

In der zweiten Welle wird angestrebt, die Auslastung im gesamten Konzern zu optimieren, um möglichst alle OP-Kapazitäten zu nutzen. Patienten, Chirurgen und Material folgen verfügbaren OP-Kapazitäten im Konzern über alle Standorte (siehe Abb. 2).

\section{Stationäre Covid-Fälle}

In dieser Kerngraphik erfassen wir alle Covid-Patientinnen und -Patienten im Haus, sowohl die Verdachtsals auch die bestätigten Fälle. Wir unterteilen diese nach Versorgungsstufe (Beatmung bis zu stationärem Pflegebedarf ohne weitere invasive Massnahmen). Die Anzahl der Beatmeten dient zur Abschätzung der be- nötigten stationären, der ambulanten sowie der intensivmedizinischen Kapazitäten. Im Verlauf der ersten Pandemiewelle konnten wir das Aufflackern von Clustern beobachten und darauf mittels variabler Intensivkapazität reagieren.

Diese Daten werden an allen Standorten erhoben und dienen zur Abschätzung der Versorgungsmöglichkeiten im Spitalverbund, ebenfalls auf Tagesbasis (siehe Abb. 3).

\section{Labor}

Alle Labor-PCR-Befunde von Covid-19 im Konzern werden erfasst und spiegeln die klinische Situation wider. Anhand der Ratio positiver Werte zum Total kann die Erkrankungswelle abgeschätzt werden. Die Positivitätsrate und deren zeitliche Änderung sind ein Anhaltspunkt für die mögliche klinische Belastung, welche mit Verzögerung eintritt (siehe Abb. 4).

\section{Datenbasierte Führung bewährt sich}

Mit der Einführung eines klaren und in der bestehenden IT-Struktur (integriertes Klinikinformationssystem der Firma Epic) einfach zu etablierenden Monitorings waren und sind wir in der Lage, unseren Betrieb von Tag zu Tag besser zu verstehen und klinische und strategische Entscheidungen datenbasiert zu treffen. Dies ist eine zur Lenkung des gesamten Konzerns relevante Ergänzung zu mathematischen Modellen sowie den Vorgaben des Regulators [5]. So zeigte sich sehr schnell, dass die zweite Welle ein neues Muster aufweist. Methodisch stehen uns aktuell die Verfahren 


\section{Laborübersicht Covid-Tests}

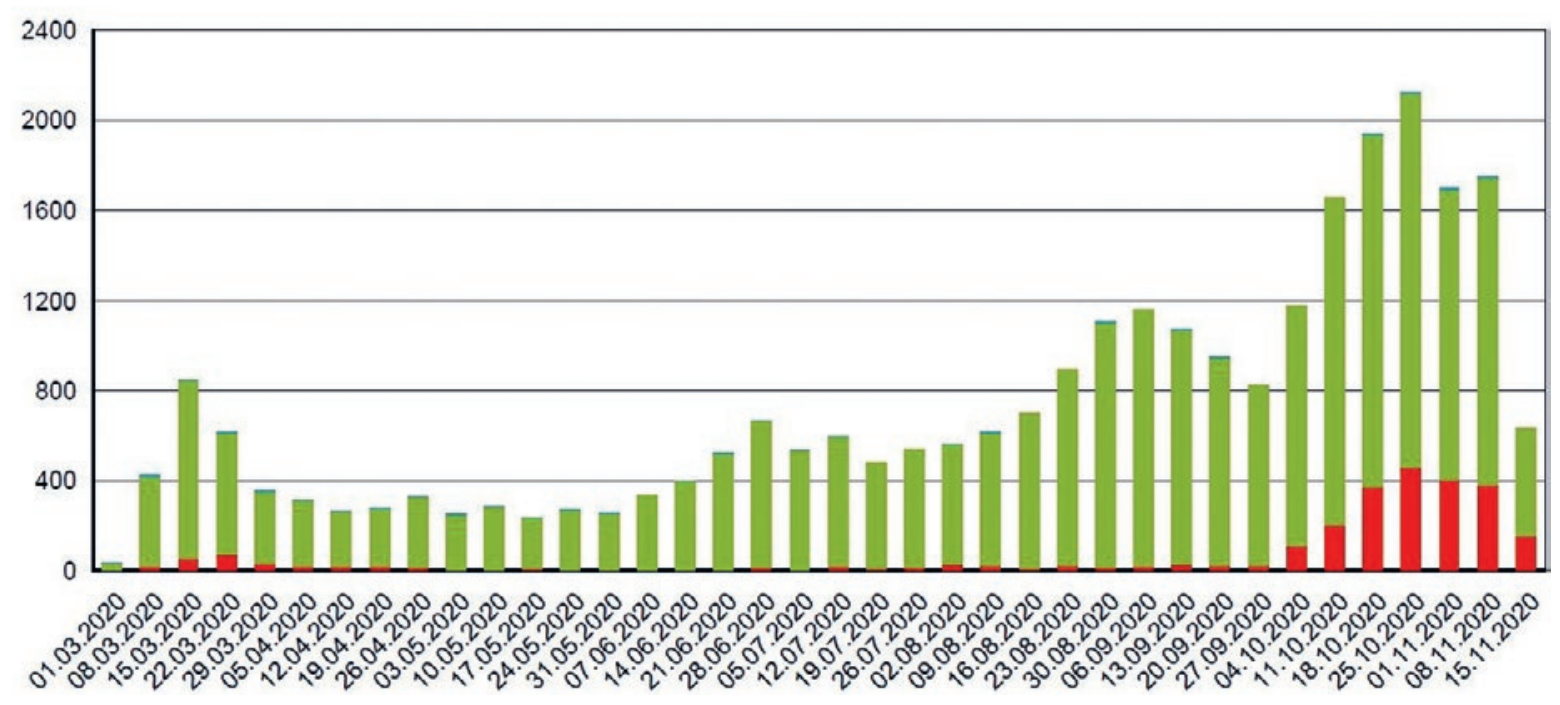

Abbildung 4: Anzahl aller Covid-Tests, März bis November 2020.

der deskriptiven Statistik zur Verfügung, die in stabileren Prozessphasen durch prädiktive Modelle ergänzt werden können.

Datenbasierte Führung hat sich in Zeiten grösster Unsicherheit bewährt. Wir konnten so in unserem Konzern bislang eine komplette Reduktion des OPProgramms vermeiden. Es ist jederzeit möglich, die etwaigen freien Personalressourcen zu erfassen und so die Allokation zu verbessern. Wenn wir einen Anstieg der benötigten Beatmungsbetten erfassen, wird Personal dorthin verteilt, wenn jedoch ein «Aufatmen» zu erkennen ist, wird dieses an anderer Stelle eingesetzt, um auch kurzfristig OP-Slots zu ermöglichen.

Konkret bilden die beschriebenen Graphiken so die Grundlage von Entscheidungen im Führungsstab be- züglich Anpassungen der elektiven und planbaren Leistungen.

Ergänzt wird dies durch Präsenz der Entscheidungsträger an den Schnittstellen im Betrieb.

\section{Literatur}

1 Osterloh F. Intensivbetten: Die Kapazitäten schwinden. Dtsch Arztebl. 2020;117.

2 Bawden D, Robinson L. The dark side of information: overload, anxiety and other paradoxes and pathologies. Journal of Information Science. 2009;35:180-91.

3 Ayres P, Paas F. Cognitive Load Theory: New Directions and Challenges. Applied Cognitive Psychology. 2012;26:827-32.

4 Vessey I. Cognitive Fit: A Theory-Based Analysis of the Graphs Versus Tables Literature. Decision Sciences. 1991;22:219-40.

5 Römmele C, Neidel T, Heins J, et al. Bed capacity management in times of the COVID-19 pandemic: A simulation-based prognosis of normal and intensive care beds using the descriptive data of the University Hospital Augsburg. Anaesthesist. 2020;69:717-25.
Prof. Dr. med. Christoph Konrad Departement für Klinische Querschnittsmedizin und Klinik für Anästhesie Tel. 0412054900 christoph.konrad[at]luks.ch

\section{Das Wichtigste in Kürze}

- Etabliere ein datenbasiertes Entscheidungsinstrument, um den Verlauf der Pandemie zu verstehen.

- Kenne deine Ressourcen in den Bereichen Technik, Logistik und - aufTagesbasis - vor allem im Bereich Personal.

- Zentralisiere möglichst alle Eingriffe und nutze verfügbare OP-Kapazität regional.

- Nutze die Positivitätsrate als Indikator für die Dimension der Behandlungslast, welche mit einer zeitlichen Verzögerung auftreten kann.

- Kommuniziere die Daten und mache dadurch Entscheidungen intern und extern verständlich.

\section{L'essentiel en bref}

- Mettez en place un outil de prise de décision basé sur des données afin de comprendre comment la pandémie se développe.

- Connaissez vos ressources au niveau de la technologie, de la logistique et, au quotidien, avant tout du personnel.

- Centralisez autant que possible les interventions et utilisez les capacités disponibles au niveau régional.

- Utilisez le taux de positivité comme indicateur de l'ampleur de la charge de travail, qui peut survenir avec un certain décalage.

- Communiquez les données afin que vos décisions soient compréhensibles à l'interne et à l'externe. 